\title{
ON THE ASYMMETRY OF STARS AT INFINITY
}

\author{
KEITH JONES AND GREGORY A. KELSEY
}

\begin{abstract}
Given a bordified space, Karlsson defines an incidence geometry of stars at infinity. These stars and their incidence are closely related to wellunderstood objects when the space is hyperbolic, CAT(0), or a bounded convex domain with the Hilbert metric. A question stemming from Karlsson's original paper was whether or not the relation of one boundary point being included in a star of another boundary point is symmetric. This paper provides an example demonstrating that this relation in the star boundary of the three-tree DiestelLeader graph $D L_{3}(q)$ is not symmetric. In doing so, some interesting bounds on distance in Diestel-Leader graphs are utilized.
\end{abstract}

\section{INTRODUCTION}

In [5], Karlsson presents a theory on the dynamics of isometries and semicontractions of metric spaces in which he develops and utilizes the idea of "stars at infinity" around boundary points of bordified metric spaces, which essentially extend the notion of half-space to the boundary of the space. For example in a CAT(0) space, the star of a boundary point is the closed ball of radius $\pi / 2$ in the angular metric. In a hyperbolic space, stars are singleton boundary points.

For a metric space $X$ with boundary $\partial X$, the star of a boundary point $\eta$ is denoted $S(\eta)$. Karlsson notes in Section 2.1 of [5] that, for $\eta, \xi \in \partial X$, it is unclear whether, or under what conditions, $\xi \in S(\eta)$ implies $\eta \in S(\xi)$; i.e., whether the relation of being included in the star is symmetric. In this paper, we exhibit an example in which this relation is not symmetric by studying the horofunction boundary of the Diestel-Leader graph $D L_{3}(q)$ (to be introduced in $\S 3$ ), which is a Cayley graph of a kind of generalization of the lamplighter group $L_{2}$. It should be noted that this example lives outside the context of non-positively curved spaces.

\section{Stars at Infinity}

2.1. Background. Karlsson introduces the following ideas in [5]. Let $\left(X, x_{0}\right)$ be a based metric space.

Definition 2.1. The halfspace for any $W \subset X$ with constant $C \geq 0$ is given by:

$$
H(W, C)=\left\{z \mid d(z, W) \leq d\left(z, x_{0}\right)+C\right\} .
$$

2010 Mathematics Subject Classification. Primary 20F65, 20F69; Secondary 20E22, 05C25.

Key words and phrases. stars at infinity, horofunction, horoboundary, Diestel-Leader graphs, lamplighter groups.

The authors would like to thank the Institute for Advanced Study for its hospitality at the Park City Math Institute Summer Session 2012, where began conversations ultimately leading to this work. 
For any bordification $\partial X$ of $X$, and any $\xi \in \partial X$ with neighborhood basis $\mathscr{U}$, the star of $\xi$ is given by:

$$
S(\xi)=\overline{\bigcup_{C \geq 0} \bigcap_{U \in \mathscr{U}} \overline{H(U, C)}}
$$

This is independent of choice of basepoint for $X$ and of neighborhood basis for $\xi$. One can also consider the star of $\xi$ based at $x_{0}$, defined by:

$$
S^{x_{0}}(\xi)=\bigcap_{U \in \mathscr{U}} \overline{H(U, 0)}
$$

and note that $S^{x_{0}}(\xi) \subseteq S(\xi)$.

Bridson \& Haefliger provide an introduction to the horofunction boundary of a metric space in [2], 8.12. We provide a brief overview here. Any based metric space $\left(X, x_{0}\right)$ has a natural embedding into the space $\mathscr{C}_{0}(X)$ of continuous functions $X \rightarrow \mathbb{R}$ with $f\left(x_{0}\right)=0$, via the mapping $x \in X \mapsto f_{x}(z)=d(x, z)-d\left(x, x_{0}\right)$. We give $\mathscr{C}_{0}(X)$ the compact-open topology and consider the closure $\bar{X}$ of $X$ in this space. This closure is compact when $X$ is proper, as is the space $\partial X=\bar{X} \backslash X$, which we call the horofunction boundary of $X$.

Definition 2.2. The horofunction $f: X \rightarrow \mathbb{R}$ defined by a sequence $\left(x_{n}\right)$ is given by:

$$
f(z)=\lim d\left(x_{n}, z\right)-d\left(x_{n}, x_{0}\right) .
$$

If $\left(x_{n}\right)$ lies along a geodesic ray with $d\left(x_{n}, x_{0}\right)=n$, we call the induced horofunction a Busemann function.

2.2. A lemma about star-inclusion. We make the following observation about stars.

Lemma 2.3. Let $X$ be a bordified metric space with basepoint $x_{0}$ and boundary $\partial X$. Let $\left(x_{n}\right)$ and $\left(y_{n}\right)$ be sequences approaching points $\bar{x}$ and $\bar{y}$, respectively, in $\partial X$. If for each $n, d\left(x_{n}, y_{n}\right) \leq d\left(x_{n}, x_{0}\right)$, then $\bar{x} \in S^{x_{0}}(\bar{y}) \subseteq S(\bar{y})$.

Proof. Let $\left\{N_{k}\right\}$ be any neighborhood basis about $\bar{y}$. Fix $k$. Then, since $y_{n} \rightarrow \bar{y}$, there exists a subsequence $\left(s_{n}\right)$ of $\left(y_{n}\right)$ contained entirely in $N_{k}$, and $s_{n} \rightarrow \bar{y}$. Let $\left(t_{n}\right)$ be the corresponding subsequence of $\left(x_{n}\right)$ (i.e., matching indices with $s_{n}$ ), so that for each $n, d\left(t_{n}, s_{n}\right) \leq d\left(t_{n}, x_{0}\right)$ and $t_{n} \rightarrow \bar{x}$. Then

$$
\left(s_{n}\right) \subseteq N_{k} \Longrightarrow\left(t_{n}\right) \subseteq H\left(N_{k}, 0\right) \Longrightarrow \bar{x} \in \overline{H\left(N_{k}, 0\right)} .
$$

Since $k$ was arbitrary, $\bar{x} \in S^{x_{0}}(\bar{y})$. Recall, $S^{x_{0}}(\bar{y}) \subseteq S(\bar{y})$.

\section{The Diestel-Leader Graph}

\subsection{Background.}

Definition 3.1 (The graph $D L_{d}(q)$ ). Let $T$ be a regular $q+1$ valent tree, such that each vertex $v$ has a single predecessor and $q$ successors. We think of successors as lying above predecessors. Let each edge have length 1 , and label the edges of $T$ so that for each vertex $v$, the $q$ successors of $v$ have labels in one-to-one correspondence with the set $\{0,1, \ldots, q-1\}$. Choose a basepoint $o$ in $T$. For $v, w \in T$, let $v \curlywedge w$ denote the greatest common ancestor of $v$ and $w$ in $T$. Define the following functions $T \rightarrow Z:$

$$
l(v)=d(v, o \curlywedge v), \quad m(v)=d(o, o \curlywedge v), \text { and } \quad h(v)=l(v)-m(v) .
$$




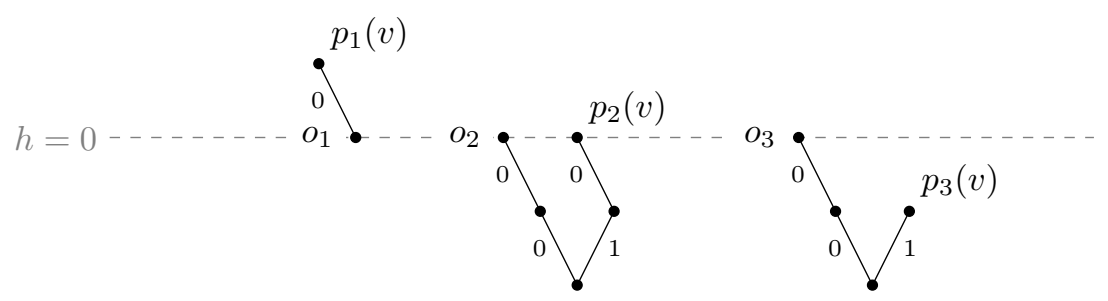

Figure 1. A point $v \in D L_{3}(2)$ having: $m_{1}=0, l_{1}=1, m_{2}=$ $l_{2}=2, m_{3}=2, l_{3}=1$.

The function $h$ gives the height in $T$, but we will make heavy use of $m$ and $l$ as well, as they appear in the distance formula provided by Stein and Taback in [6].

For a positive integer $d$, let $\left\{T_{i} \mid 1 \leq i \leq d\right\}$ be a set of copies of $T$ with basepoints $o_{i}$ and functions $m_{i}, l_{i}$, and $h_{i}$. Let $D L_{d}(q)$ be the graph whose vertices are the $d$-tuples $v=\left(v_{1}, v_{2}, \ldots, v_{d}\right), v_{i} \in T_{i}$, satisfying $\sum_{i=1}^{d} h_{i}\left(v_{i}\right)=0$. Two vertices $v$ and $w$ in $D L_{d}(q)$ are joined by an edge if there are $i \neq j$ such that: (i) $v_{i}$ and $w_{i}$ are adjacent in $T_{i}$, (ii) $v_{j}$ and $w_{j}$ are adjacent in $T_{j}$, and (iii) for all $k \notin\{i, j\}, v_{k}=w_{k}$. That is, two vertices in $D L_{d}(q)$ are adjacent if you can get from one to the other by simultaneously moving up in one tree and down in another. The graph $D L_{d}(q)$ has basepoint $o=\left(o_{1}, o_{2}, \ldots, o_{d}\right)$; since we are interested in cases where $D L_{d}(q)$ is the Cayley graph of a group, we refer to $o$ as $i d$. There are natural projections $p_{i}: D L_{d}(q) \rightarrow T_{i}$ sending $v$ to $v_{i}$. From here out, we will use $p_{i}(v)$ in lieu of $v_{i}$. We will reserve the notation $d(v, w)$ for distance between two vertices in $D L_{d}(q)$, and we will use $d_{i}\left(p_{i}(v), p_{i}(w)\right)$ to refer to the distance from the projection $p_{i}(v)$ to $p_{i}(w)$ in $T_{i}$.

Notice that for $v \in D L_{d}(q)$, since $\sum h_{i}(v)=0$, we have $\sum l_{i}(v)=\sum m_{i}(v)$; each point $v$ is determined doing the following for each tree $T_{i}$ : first select the value $m_{i}(v)$, which represents moving downward in $T_{i}$ to the height $-m_{i}(v)$, and then select a path upwards from that point that does not backtrack having length $l_{i}(v)$. This upward path corresponds to an ordered tuple in $\{0,1, \ldots, q-1\}^{l_{i}(v)}$. Figure 1 illustrates an example element of $D L_{3}(2)$.

The graph $D L_{d}(q)$ is a special case of a more general graph, $D L\left(q_{1}, q_{2}, \ldots, q_{d}\right)$ built from $d$ trees having possibly different valences; all of these are called DiestelLeader graphs after the construction in [3] of an example of a vertex-symmetric graph that they conjectured (in response to the question by Woess) is not quasiisometric to the Cayley graph of any group. Eskin, Fisher, and Whyte later proved in [4] that when $m \neq n$, this is indeed the case for $D L(m, n)$. In this paper, we will only discuss $D L_{3}(q)$, which in Corollary 3.15 of [1] is shown to be a Cayley graph of a certain affine matrix group over $\mathbb{Z} / 3 \mathbb{Z}$ with respect to a certain finite generating set.

Note: Throughout this paper, we are really interested in the vertex set of $D L_{d}(q)$, representing the corresponding group with the metric structure provided by the edges of the graph. Thus we abuse notation and use $D L_{d}(q)$ to denote the discrete group.

Definition 3.2. Let $x$ and $y$ be vertices of $\operatorname{DL}_{d}(q)$. For $1 \leq i \leq d$, we extend the $m, l$ notation to define $m_{i}(x, y)=d_{i}\left(p_{i}(x), p_{i}(x) \curlywedge p_{i}(y), l_{i}(x, y)=d_{i}\left(p_{i}(y), p_{i}(x) \curlywedge\right.\right.$ 

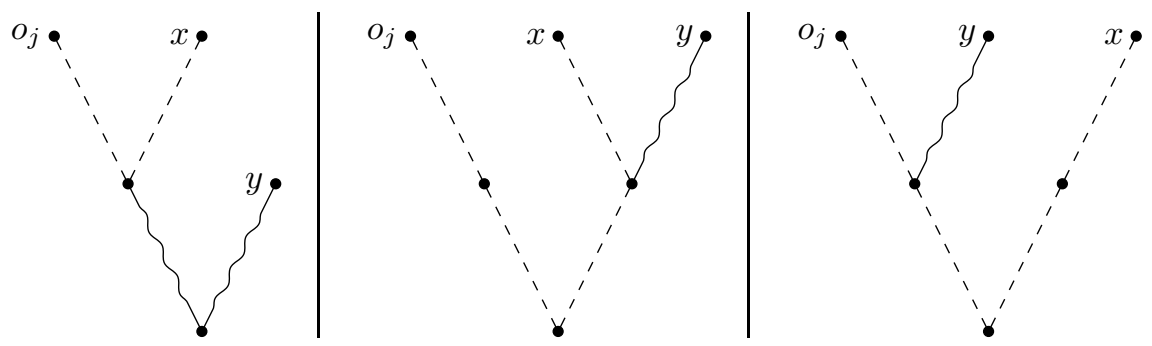

Figure 2. Schematics for the cases for Lemma 3.3.

$p_{i}(y)$, and $h_{i}(x, y)=l_{i}(x, y)-m_{i}(x, y)$. Notice that $m_{i}(y)=m_{i}(i d, y)$ and $l_{i}(y)=$ $l(i d, y)$, and $h_{i}(x, y)=h_{i}(y)-h_{i}(x)$.

Lemma 3.3. The formulas for $m_{i}(x, y)$ and $l_{i}(x, y)$ are determined by whether $m_{i}(x)$ is less than, equal to, or greater than $m_{i}(y)$, as follows:

$$
\begin{aligned}
m_{i}(x)<m_{i}(y): & m_{i}(x, y)=l_{i}(x)+\left(m_{i}(y)-m_{i}(x)\right)=m_{i}(y)+h_{i}(x) \\
& l_{i}(x, y)=l_{i}(y) \\
m_{i}(x)=m_{i}(y): & \text { set } D_{i} \text { to the length of the common upward path } \\
& m_{i}(x, y)=l_{i}(x)-D_{i} \\
& l_{i}(x, y)=l_{i}(y)-D_{i} \\
m_{i}(x)>m_{i}(y): & m_{i}(x, y)=l_{i}(x) \\
& l_{i}(x, y)=l_{i}(y)+\left(m_{i}(x)-m_{i}(y)\right)=m_{i}(x)+h_{i}(y)
\end{aligned}
$$

Proof. The schematics for each case are illustrated in Figure 2.

Definition 3.4. Let $x$ and $y$ be vertices in $\operatorname{DL}_{d}(q)$ and let $\sigma \in \Sigma_{d}$, the symmetric group on $d$ letters. For $2 \leq i \leq d-1$, define

$$
f_{\sigma, i}(x, y)=m_{\sigma(1)}(x, y)+\cdots+m_{\sigma(i)}(x, y)+l_{\sigma(i)}(x, y)+\cdots+l_{\sigma(d)}(x, y),
$$

and

$$
f_{\sigma, d}(x, y)=2 m_{\sigma(1)}(x, y)+m_{\sigma(2)}(x, y)+\cdots+m_{\sigma(d)}(x, y)+l_{\sigma(d)}(x, y)
$$

Stein and Taback derive the following distance formula in [6] (see Lemma 1 and following discussion, as well as the proof of Corollary 10) in the case that $D L_{d}(q)$ is the Cayley graph of a group.

Theorem 3.5 (Stein-Taback). Let $x$ and $y$ be vertices in $D L_{d}(q)$. For $\sigma \in \Sigma_{d}$, let $f_{\sigma}(x, y)=\max _{2 \leq i \leq d}\left\{f_{\sigma, i}(x, y)\right\}$. Then $d(x, y)=\min _{\sigma \in \Sigma_{d}}\left\{f_{\sigma}(x, y)\right\}$.

3.2. Distance bounds in Diestel-Leader graphs. One can find a variety of lower bounds on the distance between two points in $D L_{d}(q)$.

Observation 3.6. Let $R>0$. Let $x, y \in D L_{d}(q)$, and that suppose for each $\sigma \in \Sigma_{d}$, there is $i \in\{2, \ldots, d\}$ such that $f_{\sigma, i}(x, y) \geq R$. Then $d(x, y) \geq R$.

Observation 3.7. Let $x, y \in D L_{d}(q)$, and let $R=\max \left\{d_{i}\left(p_{i}(x), p_{i}(y)\right) \mid 1 \leq i \leq\right.$ $d\}$. Then $d(x, y) \geq R$. 
Lemma 3.8. Let $x, y, z \in D L_{d}(q)$ and $k \geq 0$. Suppose that for all $\sigma \in \Sigma_{d}$ and all $i \in\{2, \ldots, d\}, f_{\sigma, i}(x, z) \geq f_{\sigma, i}(x, y)+k$. Then $d(x, z) \geq d(x, y)+k$. The same is true when the inequalities are not strict.

Proof. For each $\sigma \in \Sigma_{d}$,

$$
f_{\sigma}(x, z)=\max _{2 \leq i \leq d}\left\{f_{\sigma, i}(x, z)\right\} \geq \max _{2 \leq i \leq d}\left\{f_{\sigma, i}(x, y)\right\}+k=f_{\sigma}(x, y)+k .
$$

So,

$$
d(x, z)=\min _{\sigma \in \Sigma_{d}}\left\{f_{\sigma}(x, z)\right\} \geq \min _{\sigma \in \Sigma_{d}}\left\{f_{\sigma}(x, y)\right\}+k=d(x, y)+k .
$$

Lemma 3.9. Let $x, y, z \in D L_{d}(q)$ and suppose there are nonnegative numbers $c_{j}$, $1 \leq j \leq d$, such that $m_{j}(x, z) \geq m_{j}(x, y)+c_{j}$ and $l_{j}(x, z) \geq l_{j}(x, y)+c_{j}$. Then $d(x, z) \geq d(x, y)+\sum_{j=1}^{d} c_{j}$.

Proof. Investigating Definition 3.4, we see that for each $\sigma \in \Sigma_{d}$ and $2 \leq i \leq d$, $f_{\sigma, i}(x, z)$ is a sum that can be decomposed into $d$ terms, one for each tree, such that for $1 \leq j \leq d$, tree $T_{j}$ contributes exactly one of: $m_{j}(x, z), 2 m_{j}(x, z), l_{j}(x, z)$, or $m_{j}(x, z)+l_{j}(x, z)$. Note that the term contributed depends only on $\sigma$ and $i$ and does not depend on $x$ or $z$.

The assumption that $m_{j}(x, z) \geq m_{j}(x, y)+c_{j}$ and $l_{j}(x, z) \geq l_{j}(x, y)+c_{j}$ for each $j$ ensures that $f_{\sigma, i}(x, z) \geq f_{\sigma, i}(x, y)+\sum_{j=1}^{d} c_{j}$. By Lemma 3.8, $d(x, z) \geq$ $d(x, y)+\sum_{j=1}^{d} c_{j}$.

The next results deal with points $z$ that have $h_{i}(z)=0$ for each $i$. Such points are special because $h_{i}(z)=0$ implies that $m_{i}(z)=l_{i}(z)$, which can be useful in understanding distance. Also, since $i d$ is such a point, it is easier to compare distances to these points with distances to $i d$.

Lemma 3.10 (Cases where $d(x, z) \leq d(x, i d))$. Let $x, z \in D L_{d}(q)$ and suppose $h_{i}(z)=0$ for $1 \leq i \leq d$.

(i) If $m_{i}(z)<m_{i}(x)$ for each $i$ such that $m_{i}(x) \neq 0$, and $m_{i}(z)=0$ for each $i$ such that $m_{i}(x)=0$, then $d(x, z)=d(x, i d)$.

(ii) If $m_{i}(z) \leq m_{i}(x)$ for each $i$, then $d(x, z) \leq d(x, i d)$.

Proof. We apply Lemma 3.3, where we establish the parameter $D_{i}$ for path overlap in cases where $m_{i}(z)=m_{i}(x)$.

For part (i), if $m_{i}(x) \neq 0, m_{i}(z)<m_{i}(x)$ implies $m_{i}(z, x)=m_{i}(x)+h_{i}(z)=$ $m_{i}(x)$ and $l_{i}(z, x)=l_{i}(x)$. If $m_{i}(x)=0$, the path overlap $D_{i}=0$ since $l_{i}(z)=0$, so that $m_{i}(z, x)=l_{i}(z)=m_{i}(x)$ and $l_{i}(z, x)=l_{i}(x)$. Thus for each $\sigma$ and $j$, $f_{\sigma, j}(z, x)=f_{\sigma, j}(i d, x)$, so that $d(x, z)=d(x, i d)$.

For part (ii), for $i$ such that $m_{i}(x)=m_{i}(z)>0$, we replace the equations from the previous paragraph with inequalities $m_{i}(z, x)=l_{i}(z)-D_{i}=-D_{i} \leq m_{i}(x)$, and $l_{i}(z, x)=l_{i}(x)-D_{i} \leq l_{i}(x)$. By Lemma 3.9, $d(x, z) \leq d(x, i d)$.

Lemma 3.11 (Cases where $d(x, z) \geq d(x, i d)+k)$. Let $x, z \in D L_{d}(q)$ and suppose $h_{i}(z)=0$ for each $i$. Assume $m_{i}(z) \neq m_{i}(x)$ except in cases where $m_{i}(x)=0$. Let $c_{i}=\max \left\{0, m_{i}(z)-m_{i}(x)\right\}$. Then $d(x, z) \geq d(x, i d)+\sum_{i=1}^{d} c_{i}$. 
Proof. For each $i$, we apply Lemma 3.3 to consider $m_{i}(z, x)$ and $l_{i}(x, z)$ in the following cases.

If $m_{i}(z)<m_{i}(x)$, then

$$
m_{i}(z, x)=m_{i}(x)+h_{i}(z)=m_{i}(x), \quad \text { and } \quad l_{i}(z, x)=l_{i}(x) .
$$

If $m_{i}(z)=m_{i}(x)=0$, then the path overlap $D_{i}=0$ since $l_{i}(z)=0$, so that

$$
m_{i}(z, x)=l_{i}(z)-D_{i}=m_{i}(x) \quad \text { and } \quad l_{i}(z, x)=l_{i}(x)-D_{i}=l_{i}(x) .
$$

Finally, if $m_{i}(z)>m_{i}(x)$, then $m_{i}(z, x)=l_{i}(z)=m_{i}(z)=m_{i}(x)+c_{i}$ and

$$
l_{i}(z, x)=m_{i}(z)+h_{i}(x)=\left(m_{i}(x)+c_{i}\right)+\left(l_{i}(x)-m_{i}(x)\right)=l_{i}(x)+c_{i} .
$$

By Lemma 3.9, $d(x, z) \geq d(x, i d)+\sum_{i=1}^{d} c_{i}$.

\section{Horofunctions And Stars in $D L_{d}(q)$}

\section{1. $m$-invariance of horofunctions.}

Definition 4.1 (The point $\left.\zeta_{k}^{i}\right)$. For $1 \leq i \leq d$ and $k>0$, let $\zeta_{k}^{i} \in D L_{d}(q)$ be the point given by $m_{i}\left(\zeta_{k}^{i}\right)=l_{i}\left(\zeta_{k}^{i}\right)=k$, following upward edges labeled "1", and $m_{j}\left(\zeta_{k}^{i}\right)=l_{j}\left(\zeta_{k}^{i}\right)=0$ for $j \neq i$.

Lemma 4.2. Let $x_{n}$ be a sequence defining a horofunction $h_{x}$. Then for each $1 \leq i \leq d, \lim m_{i}\left(x_{n}\right)$ exists in $\mathbb{Z}_{\geq 0} \cup\{\infty\}$.

Proof. Suppose for some $i$ that $\lim m_{i}\left(x_{n}\right)$ does not exist in $\mathbb{Z}_{\geq 0} \cup\{\infty\}$. Then since $m_{i}$ takes nonnegative integer values, there exist two subsequences $\left(y_{n}\right)$ and $\left(z_{n}\right)$ of $\left(x_{n}\right)$, both approaching $h_{x}$, such that $m_{i}\left(y_{n}\right)$ is a constant $m_{y}$ and $m_{i}\left(z_{n}\right)$ and is bounded below by $m_{y}+1$.

Let $k=m_{y}+1$. Lemma 3.11 gives $d\left(y_{n}, \zeta_{k}^{i}\right)>d\left(y_{n}, i d\right)$ for each $n$, implying $h_{x}\left(\zeta_{k}^{i}\right)>0$. But by Lemma $3.10, d\left(z_{n}, \zeta_{k}^{i}\right) \leq d\left(z_{n}, i d\right)$ for each $n$, implying $h_{x}\left(\zeta_{k}^{i}\right) \leq$ 0 , which is a contradiction.

The proof of Lemma 4.2 also proves:

Theorem 4.3 ( $m_{i}$-Invariance). If for sequences $\left(y_{n}\right)$ and $\left(z_{n}\right)$ defining horofunctions $h_{y}$ and $h_{z}$, there is an index $i$ such that $\lim m_{i}\left(y_{n}\right) \neq \lim m_{i}\left(z_{n}\right)$, then $h_{y} \neq h_{z}$.

In light of Theorem 4.3, for a horofunction $h$, we extend our notation to let $m_{i}(h)=\lim m_{i}\left(x_{n}\right)$ for any $\left(x_{n}\right)$ approaching $h$.

Corollary 4.4. If a sequence $\left(h_{n}\right)$ of horofunctions converges to a horofunction $h$, then $m_{i}\left(h_{n}\right)$ converges to $m_{i}(h)$ for each $i$.

Proof. For each $z \in D L_{d}(q)$, the sequence $\left(h_{n}(z)\right)$ must become constant on $z$ for large enough $n$, since the codomain, $\mathbb{Z}$, is discrete. By $m$-invariance, as in the proof of Lemma 4.2, if there was any $i$ such that $m_{i}\left(h_{n}\right)$ did not also eventually become constant, there would exist $z$ for which that did not occur. 


\begin{tabular}{|c|c|c|c|c|}
\hline$\sigma$ & $i$ & $f_{\sigma, i}$ & coeff. of $n$ & $f_{\sigma}$ \\
\hline$(1)$ & 2 & $m_{1}+m_{2}+l_{2}+\left(n+h_{3}\right)$ & 1 & \\
& 3 & $2 m_{1}+m_{2}+n+\left(n+h_{3}\right)$ & 2 & $2 n+2 m_{1}+m_{2}+h_{3}$ \\
\hline$(12)$ & 2 & $m_{2}+m_{1}+l_{1}+\left(n+h_{3}\right)$ & 1 & \\
& 3 & $2 m_{2}+m_{1}+n+\left(n+h_{3}\right)$ & 2 & $2 n+m_{1}+2 m_{2}+h_{3}$ \\
\hline$(13)$ & 2 & $n+m_{2}+l_{2}+l_{1}$ & 1 & \\
& 3 & $2 n+m_{2}+m_{1}+l_{1}$ & 2 & $2 n+m_{1}+l_{1}+m_{2}$ \\
\hline$(23)$ & 2 & $m_{1}+n+\left(n+h_{3}\right)+l_{2}$ & 2 & $2 n+m_{1}+l_{2}+h_{3}$ \\
& 3 & $2 m_{1}+n+m_{2}+l_{2}$ & 1 & \\
\hline$(123)$ & 2 & $m_{2}+n+\left(n+h_{3}\right)+l_{1}$ & 2 & $2 n+l_{1}+m_{2}+h_{3}$ \\
& 3 & $2 m_{2}+n+m_{1}+l_{1}$ & 1 & \\
\hline$(132)$ & 2 & $n+m_{1}+l_{1}+l_{2}$ & 1 & \\
& 3 & $2 n+m_{1}+m_{2}+l_{2}$ & 2 & $2 n+m_{1}+m_{2}+l_{2}$ \\
\hline
\end{tabular}

FiguRE 3. Distance from $\beta_{n}$ to an arbitrary point $z$, when $n$ is very large relative to the parameters of $z$.

4.2. Two Horofunctions. We will demonstrate $\alpha, \beta \in \partial D L_{3}(q)$ with $\beta \in S(\alpha)$ and $\alpha \notin S(\beta)$.

Let $\left(\alpha_{n}\right)$ be the sequence such that $l_{1}\left(\alpha_{n}\right)=m_{2}\left(\alpha_{n}\right)=n$, all other $m_{i}, l_{i}=0$, and $\left(\alpha_{n}\right)$ moves upward in $T_{1}$ choosing edges labeled " 1 ". The function $\alpha(z)=$ $\lim _{n \rightarrow \infty}\left(d\left(\alpha_{n}, z\right)-n\right.$ is a Busemann function, and therefore a horofunction ([2], 8.17-8.18). While it is not difficult to calculate $\alpha$ in general, we will not need to.

Let $\left(\beta_{n}\right)$ be the sequence $m_{3}\left(\beta_{n}\right)=l_{3}\left(\beta_{n}\right)=n, m_{i}(\beta)=l_{i}(\beta)=0$ for $i=1,2$, and the path upward in $T_{3}$ always selects the " 1 " edge.

Lemma 4.5. The sequence $\left(\beta_{n}\right)$ defines a horofunction $\beta$, and

$$
\beta(z)=m_{1}+m_{2}+\min _{j=1,2}\left\{m_{j}+h_{3}, h_{j}+h_{3}, l_{j}\right\},
$$

where each $m_{i}=m_{i}(z), l_{i}=l_{i}(z), h_{i}=h_{i}(z)$.

Proof. Let $z \in D L_{3}(q)$ and denote $m_{i}(z), l_{i}(z)$, and $h_{i}(z)$ by $m_{i}, l_{i}$, and $h_{i}$ for convenience. Assume $n>m_{i}, l_{i}$ for each $i$. We have $m_{3}\left(\beta_{n}, z\right)=n, l_{3}\left(\beta_{n}, z\right)=$ $n+h_{3}$, and $m_{i}\left(\beta_{n}, z\right)=m_{i}$ and $l_{i}\left(\beta_{n}, z\right)=l_{i}$ for $i=1,2$. Figure 3 shows the various $f_{\sigma}\left(\beta_{n}, z\right)$. The only term all $f_{\sigma}$ have in common is $2 n$, but we can rewrite $l_{i}=m_{i}+h_{i}$, and after this translation all terms have an additional $m_{1}+m_{2}$ in common. This yields:

$$
d\left(\beta_{n}, z\right)=2 n+m_{1}+m_{2}+\min \left\{m_{1}+h_{3}, m_{2}+h_{3}, l_{1}, h_{2}+h_{3}, h_{1}+h_{3}, l_{2}\right\} .
$$

This yields $d\left(\beta_{n}, i d\right)=2 n$, and $\beta(z)=\lim _{n \rightarrow \infty} d\left(\beta_{n}, z\right)-2 n$.

\subsection{Neighborhoods and Stars.}

Lemma 4.6. $\beta \in S(\alpha)$.

Proof. As noted, $d\left(\beta_{n}, i d\right)=2 n$, by moving as necessary in $T_{3}$ and compensating in either $T_{1}$ or $T_{2}$. We cannot apply Lemma 4.5, as the parameters of $\alpha_{n}$ grow along with $\beta_{n}$. But we can find $d\left(\beta_{n}, \alpha_{n}\right)$ directly: we can move from $\beta_{n}$ to $\alpha_{n}$ in $2 n$ steps as well, by moving in $T_{3}$ as necessary, compensating in $T_{2}$ for the first $n$ steps 
and then in $T_{1}$ for the final $n$ steps. So $d\left(\beta_{n}, \alpha_{n}\right)=2 n=d\left(\beta_{n}, i d\right)$. By Lemma 2.3, $\beta \in S(\alpha)$.

Recall, the point $\zeta_{k}^{i}$, introduced in Definition 4.1 , has $m_{i}=l_{i}=k$, and all other parameters trivial.

Lemma 4.7. Let $z \in D L_{3}(q)$ have $m_{i}(z)>0$ for $i=1$ or 2 . Then $d\left(z, \zeta_{1}^{i}\right)-$ $d(z, i d) \neq \beta\left(\zeta_{1}^{i}\right)$.

Proof. By Lemma 3.10, $d(z, \zeta) \leq d(z, i d)$, so $d(z, \zeta)-d(z, i d) \leq 0$, while Lemma 3.11 ensures $\beta(\zeta)>0$.

Definition 4.8. For $j \in\{1,2\}, k \in \mathbb{Z}_{\geq 0}$, and $\epsilon \in\{0,1, \ldots, q-1\}$, let $\nu_{k}^{j, \epsilon}$ be the point in $D L_{3}(q)$ with $d\left(\nu_{k}^{j, \epsilon}, i d\right)=k$ obtained by moving $k$ edges (all labeled $\epsilon$ ) upward in $T_{j}$ and $k$ edges downward in $T_{3}$. So $l_{j}\left(\nu_{k}^{j, \epsilon}\right)=m_{3}\left(\nu_{k}^{j, \epsilon}\right)=k$, while all other $m_{i}, l_{i}=0$.

Lemma 4.9. Let $z \in D L_{3}(q)$ have $m_{1}(z)=m_{2}(z)=0$, and assume $l_{j}(z)>0$ for some $j \in\{1,2\}$. Choose label $\epsilon$ not equal to the first edge joining $o_{j}$ to $p_{j}(z)$. Then $\nu=\nu_{1}^{j, \epsilon}$ has $d(z, \nu)-d(z, i d) \neq \beta(\nu)$.

Proof. By Lemma 4.5, $\beta(\nu)=-1$. Lemma 3.6 will ensure that $d(z, \nu)-d(z, i d) \geq 0$ if for each $\sigma \in \Sigma_{d}$, there is at least one $i$ such that $f_{\sigma, i}(z, \nu) \geq d(z, i d)$.

For each $k=1,2,3$, denote $m_{k}(z)$ and $l_{k}(z)$ by $m_{k}$ and $l_{k}$ respectively. Assume for concreteness that $l_{1}>0$. Note that $m_{3}=\sum_{k=1}^{3} l_{k}$. Also, $d(z, i d)=$ $d_{3}\left(o_{3}, p_{3}(z)\right)=l_{1}+l_{2}+2 l_{3}$ since Observation 3.7 ensures this is a lower bound on $d(z, i d)$, and it can be realized by moving in $T_{3}$ and compensating in $T_{1}$ and $T_{2}$ as appropriate.

We have the following distances, by Lemma 3.3.

$$
\begin{array}{ccc}
m_{1}(z, \nu)=l_{1} & m_{2}(z, \nu)=l_{2} & m_{3}(z, \nu)=l_{3} \\
l_{1}(z, \nu)=1 & l_{2}(z, \nu)=0 & l_{3}(z, \nu)=\sum l_{k}-1
\end{array}
$$

The following table provides, for each $\sigma$, an $f_{\sigma, i}(z, \nu) \geq l_{1}+l_{2}+2 l_{3}$ :

\begin{tabular}{|l|l|}
\hline$f_{(1), 3}=2 l_{1}+l_{2}+l_{3}+\left(\sum l_{k}-1\right)$ & $f_{(12), 3}=2 l_{2}+l_{1}+l_{3}+\left(\sum l_{k}-1\right)$ \\
\hline$f_{(13), 3}=2 l_{3}+l_{2}+l_{1}+1$ & $f_{(23), 2}=l_{1}+l_{3}+\left(\sum l_{k}-1\right)$ \\
\hline$f_{(123), 2}=l_{2}+l_{3}+\left(\sum l_{k}-1\right)+1$ & $f_{(132), 3}=2 l_{3}+l_{1}+l_{2}$ \\
\hline
\end{tabular}

If $l_{2}>0$ instead, the calculation is symmetric.

Definition 4.10. For any finite set $F$, let

$$
B(F)=\left\{f \in \overline{D L_{3}(q)}|f|_{F}=\left.\beta\right|_{F}\right\} .
$$

and set

$$
F_{0}=\left\{\zeta_{1}^{1}, \zeta_{2}^{1}, \nu_{1}^{1,0}, \nu_{1}^{1,1}, \nu_{1}^{2,0}, \nu_{1}^{2,1}\right\}
$$

Because the functions in $\overline{D L_{3}(q)}$ map from the discrete space $D L_{3}(q)$ to the discrete space $\mathbb{Z}$, when we consider the compact-open topology on $\overline{D L_{3}(q)}$, the collection $\{B(F)\}, F$ a finite set, is a neighborhood basis for $\beta$. Thus $B\left(F_{0}\right)$ is an open set about $\beta$.

Theorem 4.11. A sequence $\left(b_{n}\right) \subset D L_{3}(q)$ approaches $\beta$ if and only if it has a tail whose projections are trivial in $T_{1}$ and $T_{2}$ and $m_{3}\left(b_{n}\right) \rightarrow \infty$. 
Proof. By Lemmas 4.7 and 4.9, no $x \in D L_{3}(q)$ that is nontrivial in $T_{1}$ or $T_{2}$ lies in $B\left(F_{0}\right)$. This proves the forward direction.

Now suppose $\left(b_{n}\right)$ is trivial in $T_{1}$ and $T_{2}$ and has $m_{3}\left(b_{n}\right) \rightarrow \infty$. Then $l_{3}\left(b_{n}\right)=$ $m_{3}\left(b_{n}\right)$. The calculations in the proof of Lemma 4.5 show that when $n$ is large relative to the parameters of $z \in D L_{3}(q)$, the choice of upward path in $T_{3}$ is irrelevant to the calculation. We can replace $n$ with $m_{3}\left(b_{n}\right)$ in that calculation, and we obtain the same result.

Corollary 4.12. The horofunction $\beta$ is isolated in $\partial D L_{3}(q)$.

Proof. Let $\left(h_{n}\right) \subset \partial D L_{3}(q)$ be a sequence of horofunctions approaching $\beta$. Then $\left(h_{n}\right)$ has a tail $\left(t_{n}\right)$ lying in $B\left(F_{0}\right)$. For each $n$, any sequence $\left(x_{k}\right) \in D L_{3}(q)$ approaching $t_{n}$ also has a tail in $B\left(F_{0}\right)$. By Theorem 4.11. $\left(t_{n}\right)$ is the constant sequence $(\beta)$.

It is worth noting that while $\beta$ is isolated in the boundary, $S(\beta) \neq\{\beta\}$. For example, let $Y \subseteq\{1,2,3\}$ contain 3 and at least one other index. Let $\left(\gamma_{n}\right)$ be any sequence with $m_{i}\left(\gamma_{n}\right)=l_{i}\left(\gamma_{n}\right)=n$ for $i \in Y$, and $m_{i}\left(\gamma_{n}\right)=l_{i}\left(\gamma_{n}\right)=0$ otherwise. It can be shown that $\left(\gamma_{n}\right)$ defines a horofunction $\gamma \neq \beta$; and one can calculate $d\left(\gamma_{n}, i d\right) \geq d\left(\gamma_{n}, \beta_{n}\right)$ for each $n$, so that Lemma 2.3 ensures $h_{\gamma} \in S(\beta)$.

Corollary 4.13. For $k \geq 0$, let

$N_{k}(\beta)=\left\{z \in D L_{3}(q) \mid m_{i}(z)=l_{i}(z)=0\right.$ for $i=1,2$ and $\left.m_{3}(z)=l_{3}(z) \geq k\right\} \cup\{\beta\}$.

Then the family $\left\{N_{k}(\beta)\right\}, k \geq 0$, is a neighborhood basis for $\beta \in \overline{D L_{3}(q)}$.

Note that $\beta_{n}$ and $\beta$ are not the only elements of $N_{k}(\beta)$, since alternate choices of edge labels may be made.

Theorem 4.14. Let $h \in \partial D L_{3}(q)$ have $m_{3}(h)=0$. Then $h \notin S(\beta)$. As a consequence, $\alpha \notin S(\beta)$.

Proof. By $m$-invariance, any sequence of points in $D L_{3}(q)$ approaching $h$ has a tail $\left(a_{n}\right)$ such that for each $n, m_{3}\left(a_{n}\right)=0$. For any $C \geq 0$, when $k>C$, the structure of $N_{k}(\beta)$ and Lemma 3.11 together imply that

$$
d\left(a_{n}, N_{k}(\beta)\right) \geq d\left(a_{n}, i d\right)+k>d\left(a_{n}, i d\right)+C .
$$

So $a_{n} \notin H\left(N_{k}(\beta), C\right)$, and so $h \notin \cap_{k>0} \overline{H\left(N_{k}(\beta), C\right)}$.

By Corollary 4.4, any sequence $\left(h_{n}\right)$ of horofunctions approaching $h$ must also eventually have $m_{3}\left(h_{n}\right)=0$ as well, so $h$ is also not a limit of any sequence of horofunctions in the union of $\bigcap_{k>0} \overline{\left.H\left(N_{k}(\beta), C\right)\right)}$ over all $C$.

Thus

$$
h \notin \overline{\bigcup_{C \geq 0}\left(\bigcap_{k>0} \overline{H\left(N_{k}(\beta), C\right)}\right)}=S(\beta) .
$$

\section{REFERENCES}

[1] Laurent Bartholdi, Markus Neuhauser, and Wolfgang Woess. Horocyclic products of trees. J. Eur. Math. Soc. (JEMS), 10(3):771-816, 2008

[2] Martin R. Bridson and André Haefliger. Metric spaces of non-positive curvature, volume 319 of Grundlehren der Mathematischen Wissenschaften [Fundamental Principles of Mathematical Sciences]. Springer-Verlag, Berlin, 1999. 
[3] Reinhard Diestel and Imre Leader. A conjecture concerning a limit of non-Cayley graphs. J. Algebraic Combin., 14(1):17-25, 2001.

[4] Alex Eskin, David Fisher, and Kevin Whyte. Coarse differentiation of quasi-isometries I: Spaces not quasi-isometric to Cayley graphs. Ann. of Math. (2), 176(1):221-260, 2012.

[5] Anders Karlsson. On the dynamics of isometries. Geom. Topol., 9:2359-2394, 2005.

[6] M. Stein and J. Taback. Metric Properties of Diestel-Leader Groups. Michigan Math. J., 62(2):365-286, 2013.

Department of Mathematics, Computer Science \& Statistics; State University of New York College at Oneonta; 108 Ravine Parkway; Oneonta, NY 13820

E-mail address: keith.jones@oneonta.edu

Department of Mathematics; Bellarmine University; 2001 Newburg Rd.; Louisville, KY 40205

E-mail address: gkelsey@bellarmine.edu 